\title{
Rheumatic Heart Disease in Children
}

\author{
Mira Govindarajan \\ ${ }^{1}$ CARE Hospitals Banjara Hills, Hyderabad, India
}

Ind J Car Dis Wom 2019;4:40-41

\begin{abstract}
Address for correspondence Mira Govindarajan, MD, MHA, Consultant Non-Interventional Cardiology, CARE Hospitals Banjara Hills Hyderabad (e-mail: miragdoc178@gmail.com).
\end{abstract}

\begin{abstract}
Keywords

- RHD in children

- RHD

- burden

This article is based on the study of girl students aged 6 to 18 years from Government Hostel, Moosarambagh, Hyderabad on July 1, 2018. The students were screened for cardiac disease clinically and echocardiographically. There were no cases of rheumatic disease. This article presents a review of trends in the prevalence of rheumatic heart disease (RHD) in the community worldwide and in India. It also presents echocardiographic criteria for screening in RHD.
\end{abstract}

\section{Introduction}

On the occasion of Doctors' day, July 1, 2018, we conducted a camp in the girl's hostel under the auspices of WINCARS. One hundred and twenty girl students between the age groups of 6 to 16 years were screened clinically and echocardiographically for the presence of cardiac disease with specific reference to rheumatic heart disease (RHD).

We were surprised to find that not even one student had the stigmata of RHD or history suggestive of rheumatic fever. We decided to look at the changing trends in the burden of disease both locally and nationally.

Global age-standardized mortality for RHD decreased by $47.8 \%$ from 1990 to 2015 , as per a landmark study published in the New England Journal of Medicine. ${ }^{1}$ Still, the burden of disease was significant in specific regions in South Asia India among them. Furthermore, India had the highest number of deaths due to RHD in 2015 with 119,000 people dying from this disease. It was also one of the five countries with the highest number of cases accounting for $73 \%$ of the global cases. Subsequent correspondence established that RHD constituted $42 \%$ of cardiac diseases in rural central India. ${ }^{2}$

As per our national statistics, a population-based study showed the prevalence of RHD to be about 2 per 1,000, while a survey conducted among school children in the age group of 5 to 16 years by the Indian Council of Medical Research (ICMR) gave an overall prevalence of 6 per 1,000 (range: $1.8-11 / 1,000){ }^{3}$ Clinical prevalence of RHD in the undivided state of Andhra Pradesh was 0.7 per 1,000, prevalence by echocardiographic criteria was 7.6 per 1,000 , and the total prevalence was 8.3 per 1,000 population. ${ }^{4}$ Despite the smaller number of children we studied, going by the age and social stratum we would expect at least one or two cases.
Rheumatic fever is endemic in India accounting for nearly 25 to $45 \%$ of acquired heart disease. The clinical spectrum as established by a study in rural India is as follows: ${ }^{5}$ arthritis, chorea, and erythema marginatum were seen in 67.78, 5.87 , and $1 \%$ cases, respectively. Overall, $1 \%$ had pericardial effusion, and $32.38 \%$ developed congestive cardiac failure. Benzathine penicillin prophylaxis was regular in $56.87 \%$ cases and $15.6 \%$ cases presented with recurrence of the disease. Positive family history was present in $7.88 \%$ of cases. ${ }^{5}$ In our camp, only a few sore throat histories were there in children without any other manifestations of rheumatic fever.

Poverty, overcrowding, poor access to health care, inadequate use of penicillin due to fear of allergy are some of the causative factors for high prevalence. The ICMR primary health centers and school health services are two levels of prevention and control. ${ }^{6}$ The children we studied came from a poor socioeconomic stratum and were living in a government-aided hostel. Even so, we could not get a single clinical case of rheumatic fever or RHD.

While echocardiography serves to detect established RHD, its use in the diagnosis of carditis needs further evaluation. ${ }^{7}$ Other limitations for echo-based diagnosis of RHD is the need for a high degree of expertise. ${ }^{8}$ In our camp, qualified cardiologists did the echocardiogram. As suggested by the World Health Federation, we took anterior mitral valve leaflet thickening greater than or equal to $3 \mathrm{~mm}$ (age-specific), chordal thickening, restricted leaflet motion, and excessive leaflet tip motion during systole of mitral valve as criteria for RHD. Similarly, for aortic valve, irregular or focal thickening, coaptation defect, restricted leaflet motion, and prolapse of the aortic valve were considered as diagnostic of RHD.
License terms

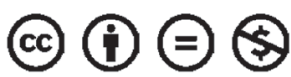


Preventative strategy would decrease the incidence, prevalence, morbidity, mortality, and severity of RHD. In India, the National Rheumatic Heart Consortium convened a diverse set of professionals dedicated to this cause. ${ }^{9,10}$

Does the discrepancy between the previous literature and the present study truly reflect decreasing incidence of rheumatic fever and RHD as the result of effective preventive strategies? We want to bring this information to the notice of all doctors who see populations traditionally at risk in order to establish whether there is a fall in the occurrence of rheumatic fever or RHD in our population.

\section{Conflict of Interest}

None declared.

\section{References}

1 Watkins DA, Johnson CO, Colquhoun SM, et al. Global, regional, and national burden of rheumatic heart disease, 1990-2015. N Engl J Med 2017;377:713-722

2 Sohrabi B, Ranjbar A. Global burden of rheumatic heart disease. Engl J Med 2018;378(1):e2

3 National Health Portal, Rheumatic Fever and Rheumatic Heart Disease. 2016. Available at:https://www.nhp.gov.in/disease/ communicable-disease/rheumatic-fever-and-rheumaticheart-disease. Accessed May 30, 2019

4 Rama Kumari N, Bhaskara Raju I, Patnaik AN, et al. Prevalence of rheumatic and congenital heart disease in school children of Andhra Pradesh, South India. J Cardiovasc Dis Res 2013;4(1):11-14

5 Ghosh S, Dey S. Clinical profile of rheumatic heart disease in children and young people in Eastern India. International Journal of Collaborative Research on Internal Medicine and Public Health 2011;2(3):100-107

6 Padmavathy S. Rheumatic heart disease: prevalence and preventive measures in the Indian subcontinent. BMJ 2001;86(2):127

7 Shah B, Sharma M, Kumar R, Brahmadathan KN, Abraham VJ, Tandon R. Rheumatic heart disease: progress and challenges in India. Indian J Pediatr 2013;80(Suppl 1):S77-S86

8 Saxena A. Task shifting rheumatic heart disease screening to non-experts. Lancet Glob Health 2016;4(6):e349-e350

9 Reményi B, Wilson N, Steer A, et al. World Heart Federation criteria for echocardiographic diagnosis of rheumatic heart disease-an evidence-based guideline. Nat Rev Cardiol 2017

10 Marijon E, Ou P, Celermajer DS, et al. Prevalence of rheumatic heart disease detected by echocardiographic screening. N Engl J Med 2007;357(5):470-476 